
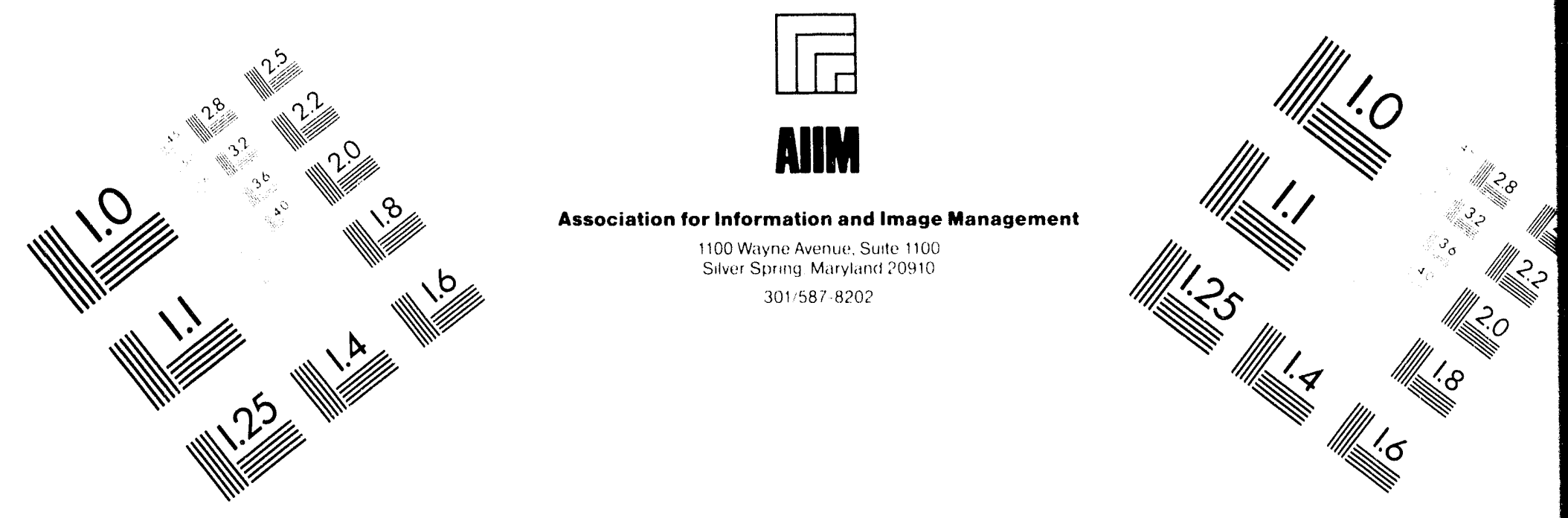

\title{
Centimeter
}

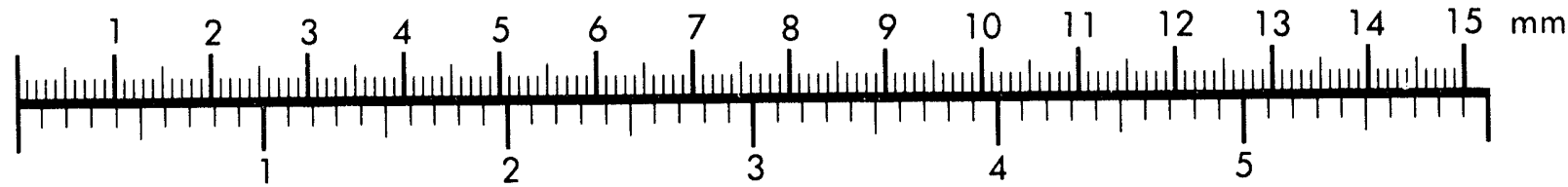

Inches
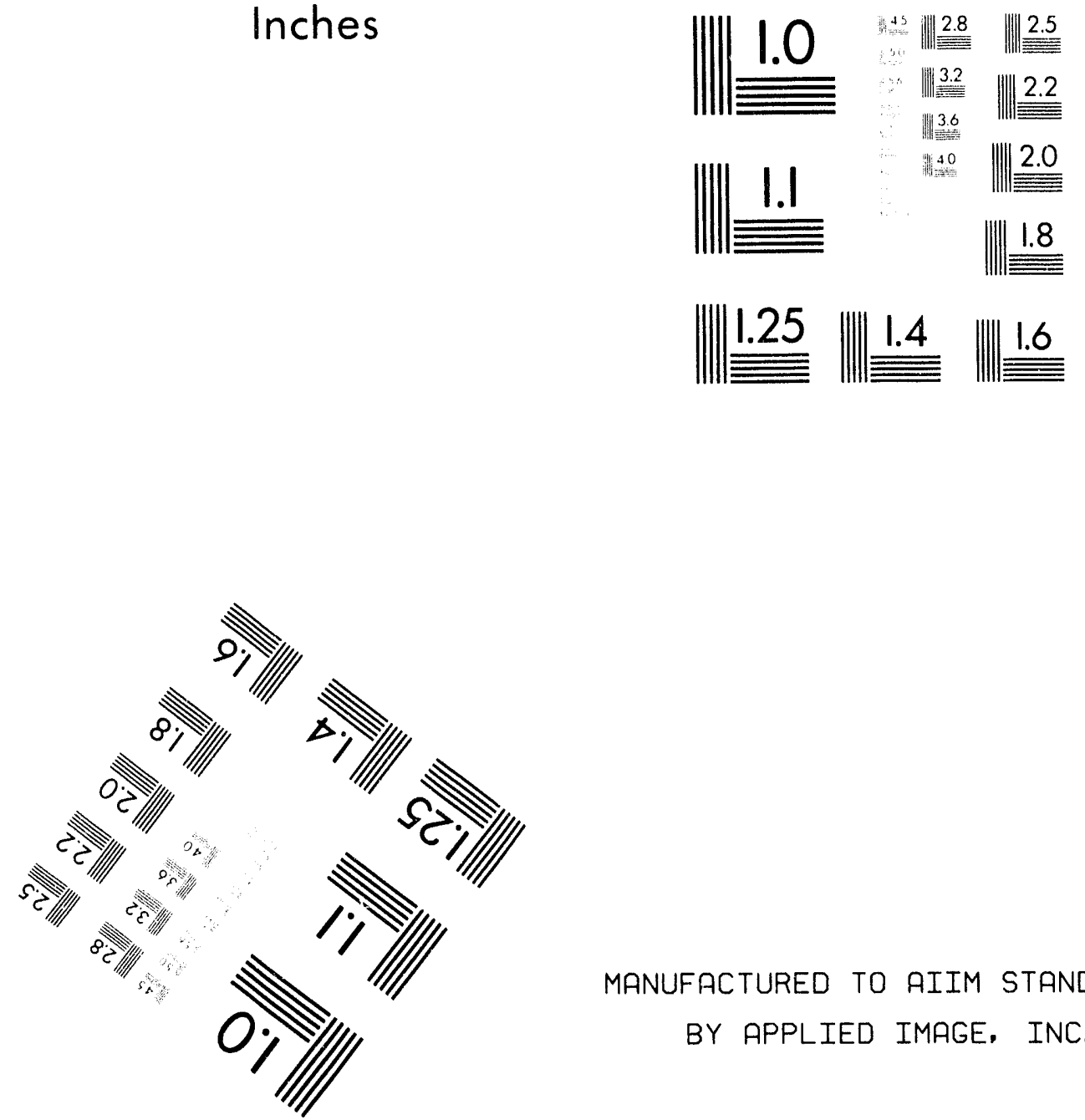

MANUFACTURED TO AIIM STANDARDS

BY APPLIED IMAGE, INC.

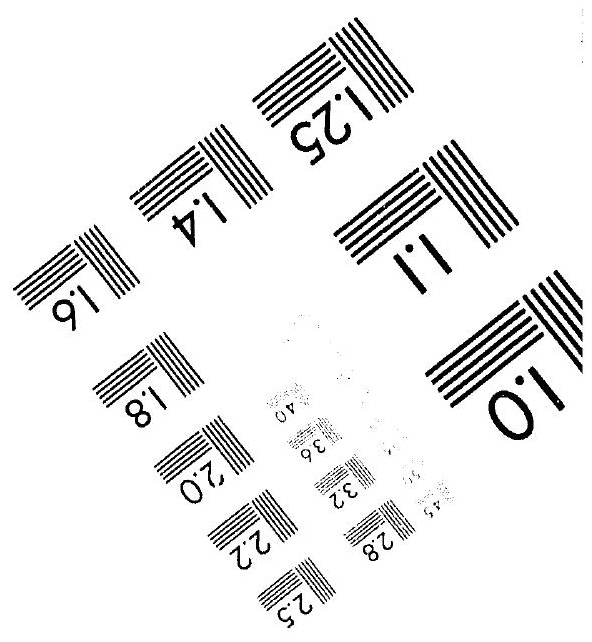



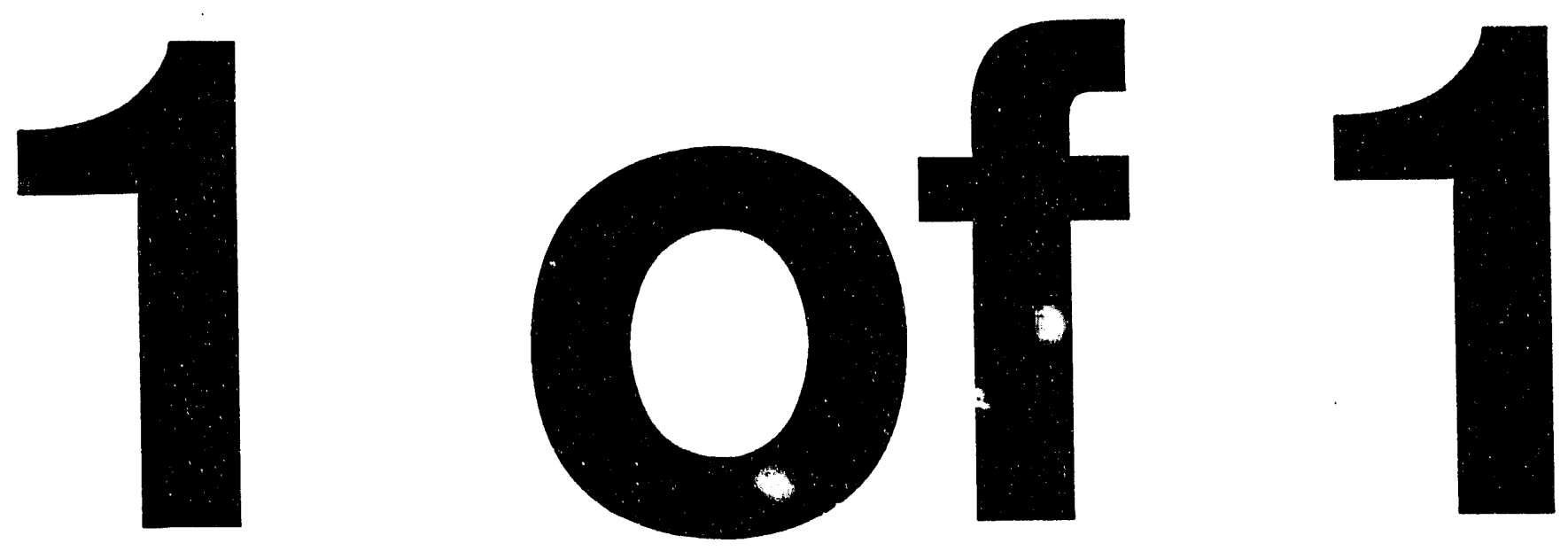


\title{
(8) $15-9405170-2$
}

\section{STUDIES OF POSITRON INDUCED LUMINESCENCE FROM POLYMERS}

\author{
JUN XU, L. D. HULETT, JR., T. A. LEWIS, AND N. H. TOLK' \\ Oak Ridge National Laboratory, P. O. Box 2008, Oak Ridge, TN 37831 \\ 'Dept. of Phys. and Astron., Vanderbilt Univ., Nashville, TN 37235
}

\begin{abstract}
Keywords: positron-induced luminescence, catholuminescence, photoluminescence, electron-hole pair, anthracene, and polymers.
\end{abstract}

Abstract: Light emission from polymers (anthracene dissolved in polystryrene) induced by lowenergy positrons and electrons has been studied. Results indicate a clear difference between optical emissions under positron and electron bombardment. The positron-induced luminescence spectrum is believed to be generated by both collisional and annihilation processes.

Positron bombardment of materials may produce light in the visible, UV, and IR reyions. There have been no spectral studies focused on optical emission induced by slow positrons. This work will address the feasibility of positron-induced luminescence (PIL) as a probe for materials analysis.

Light emission arising from electron (catholuminescence) and photon (photoluminescence) bombardment of materials has been widely used for materials analysis. In these processes, an electron in a valance band is initially pumped to a conduction band or an excited state. An electron-hole pair is formed after electron or photon bombardment. The pair develops, in some circumstances, color centers in certain insulators and semiconductors, involving the self-trapped exciton (STE) . The electron of the STE recombines with the hole, leading to light emission, as illustrated in Fig. 1(a).

For low-energy positron bombardment, light emission mechanisms may be different from those associated with electron-hole production. A positron must annihilate with an electron of the occupied bands. As a result, a single hole is produced in the band by annihilation, not an electron-hole pair. Light emission associated with electron-hole pairs is

not important if the positron energy is low. Electrons from impurities, defects, and surface states will fill the vacancy left after annihilation. Of course, light results as indicated in Fig. 1(b). Therefore positron induced luminescence (PIL) may be targeted to unique features of defects, impurities, and material structures.

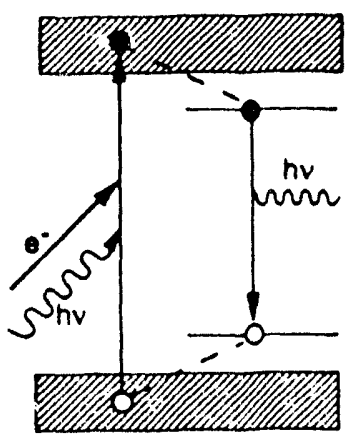

(a) Electron-Hole Pair

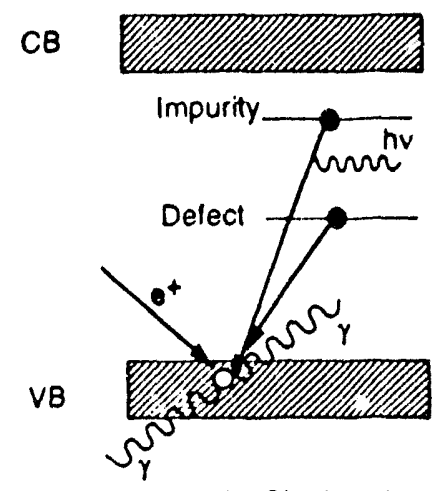

(b) Single hole

Figure 1. Energy level diagram indicating light emission due to (a) electron or photon bombardment and (b) slow positron bombardment. In (a), an electron-hole pair is produced. In (b), only a single hole is formed by positron annihilation. 


\section{DISCLAIMER}

This report was prepared as an account of work sponsored by an agency of the United States Government. Neither the United States Government nor any agency thereof, nor any of their employees, makes any warranty, express or implied, or assumes any legal liability or responsibility for the accuracy, completeness, or usefulness of any information, apparatus, product, or process disclosed, or represents that its use would not infringe privately owned rights. Reference herein to any specific commercial product, process, or service by trade name, trademark, manufacturer, or otherwise does not necessarily constitute or imply its endorsement, recommendation, or favoring by the United States Government or any agency thereof. The views and opinions of authors expressed herein do not necessarily state or reflect those of the United States Government or any agency thereof. 
Measurements were performed at the Oak Ridge Electron Linear Accelerator (ORELA) Positron Facility. The experimental setup consisted of a high vacuum chamber operated at about $7.0 \times 10^{-9}$ Torr, a positron beam line, and an optical spectrometer, as described in Ref. 1. The ORELA provides a pulsed electron beam (pulse with: 10-20 ns, beam energy: $150 \mathrm{MeV}$, repetion rate $800 \mathrm{~Hz}$, power: $3 \mathrm{~kW}$ ). The electron heam bombards a tantalum target and produces intense gamma rays. The forward scattered gamma photons are captured by 10 elements of tungsten plates that converts them to electron-positron pairs. The tungsten plates have been annealed in such a manner that they also serve as efficient moderators of the fast positrons. An extraction tube is placed immediately in front of the moderator. A negative 3-kV pulse is applied to the extraction tube for accelerating the $2-3 \mathrm{eV}$ positrons to $3 \mathrm{keV}$.

In this beam line, elections can also be extracted by timing the high voltage pulse. As stated above, a negative high-voltage pulse is applied to the end of the extraction tube for accelerating slow positrons out of the target room. A positive pulse, due to reflection, follows the negative pulse after about 40 ns. By adjusting the time of the positive reflection to coincide with the time period in which the ORELA beam is impacting the target, we can obtain a pulsed electron beam having an average current or about $10^{10}$ electrons per second. Positrons or electrons can be exclusively selected by matching the extraction pulse to the ORELA pulse time.

The samples used in these experiments are anthracene dissolved in polystyrene. A grid is mounted in front of the sample, which is electronically connected to the sample. A negative voltage is applied to the grid to reject possible electrons and to control the positron energy. A heating wire is sandwished inside the target holder to change the temperature of the sample. A chromel-alumel thermocouple is physically attached to the target to ineasure the sample temperature. This experiment was done at room temperature. A Sm-Co permanent magnet, for focusing positrons, was fixed at the rear of the target. The axial magnetic field at the sample surface is measured to be about 2,600 Gauss. A micro-channel plate is mounted on a rotatable feedthrough for monitoring the heams.

Light emitted from the sample was imaged, with a two-lens array, onto the entrance slit of a monochromator, such that the $f$-number of the source matched the f-number of the spectrometer. The first lens was biconvex and was placed such that its focal length, $38 \mathrm{~mm}$, coincided with the mid point of the sample. Light emitted from the sample was collimated and transported to a plano convex lens having a focal length of $500 \mathrm{~mm}$, the same as the distance between the entrance slit of the spectrometer to the diffraction grating. The grating of the spectrometer had 1200 lines $/ \mathrm{mm}$, with a transmission range of 2000 to $8000 \AA$. A photomultiplier tube (PMT), operated at -950 volts, was used as the detector.

Time variations of light emission were measured. The ORELA pulse served as the start signal, and the stop signal was given by the photons detected by the PMT. The time interval was recorded by a time-to-digital converter, interfaced through a computer-aided measurement-and-control (CAMAC) unit to a personal computer. Photons are generated by the initial gamma flash of the accelerator and slow positrons. The light induced by the gamma flash was the first to arrive at the detector, while that induced by the slowermoving $3 \mathrm{keV}$ positrons arrived later at the PMT. Luminescence induced by the fast and slow particles were distinguished by the time windows of their detection.

Figure 2 shows an example of the timedependant nature of the light emission that occurred after bombardment by the initial gamma flash and slow positrons. The monochromater was set at $4188 \AA$. The first peak was generated by the initial gamma ray bombardment of the target. These particles are the first to reach the target and produce light. The peak induced by the positrons having energies under $3 \mathrm{keV}$ occurs about $300 \mathrm{~ns}$ later. 
The 300 ns period is about the time required for the $3-\mathrm{keV}$ positrons travel the 10 meter flight path. The time delay of the second peak varies with the extraction voltage.

Light emission induced by the slow positron bombardment is plotted in Figure 3(a). The data are the integrated counts over the slow peak, shown in Figure 2. The spectrometer was calibrated by measuring the known He-Ne laser and Mercury lamp lines. The vertical scale is set to be unity for $4188 \AA$. The dominant band occurs in the range of

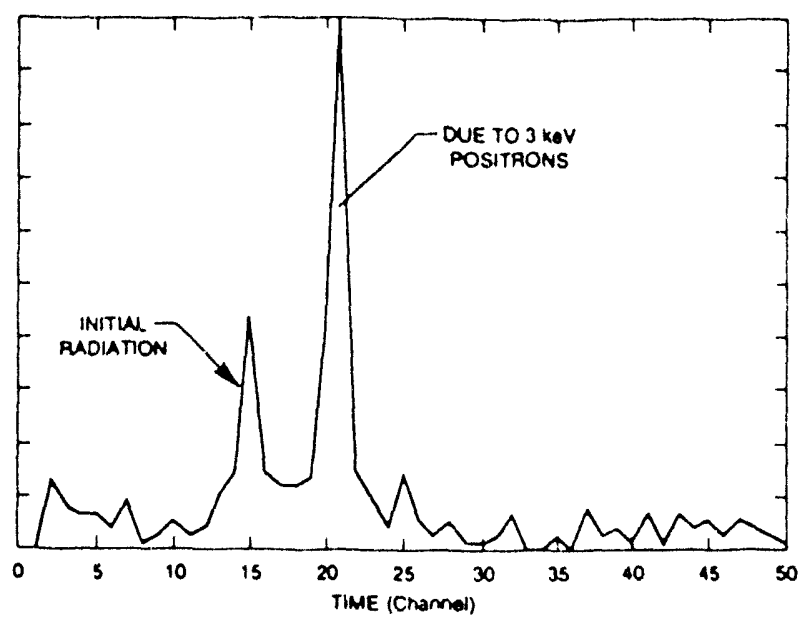

Figure 2. Light emission yield as a function of time. The wavelength was selected at $4188 \AA$.

4000-4700 $\AA$. Note the smaller band that occurs below $4000 \AA$ (sub-blue) for the positron induced spectrum; this band is absent in the electron-induced spectrum.

The optical spectrum induced from the same sample by $2 \mathrm{keV}$ electrons is plotted in Figure 3(b). Two bands are observed. The first is in range $4000-4900 \AA$, which is essentially the same as that induced by the slow positrons. The second band is broader, ranging from 4700 to $6000 \AA$. The weaker band below $4000 \AA$, induced by positrons, is not detected in the electron induced spectrum.

Figure 4 shows the time dependence of spectra for different bands under electron and positron bombardment. The pulse width for both positrons and electrons is about 40 ns, which is slightly larger than the widths of the
ORELA and the extraction pulse width. The lifetime for the blue band emission is about 70 ns. The lifetime for the yellow band emission is $130 \mathrm{~ns}$. The lifetime for sub-4000 $\AA$ band under positron bombardment is less than $60 \mathrm{~ns}$. These results show that the sub-4000 $\AA$ emission under positron bombardment and the blue band emission for both positron and electron bombardment are fast. The yellow band, which is induced only by the electron bombardment, grows more slowly and decays more slowly.

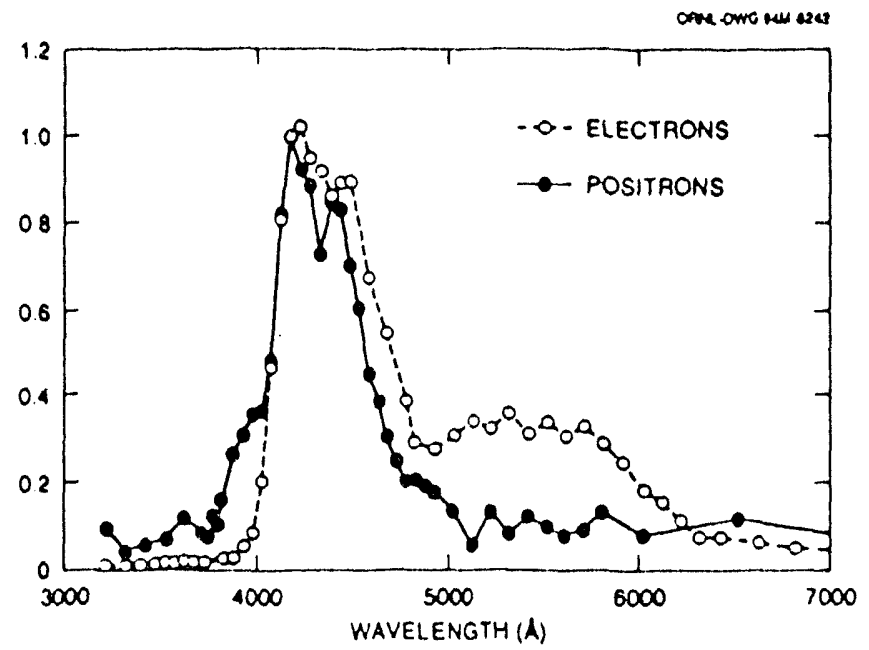

Figure 3. Optical spectra under (a) positron and (b) electron bombardment. The time windows were resolved for positrons or electrons.

Before excitation, the polymer was in a singlet ground state. Absorption of electron or positron energy excited the molecules directly to singlet electronic states and indirectly to metastable triplet states [2]. Accompanying the electron excitation, a hole is produced in the valance band. The most probable pathway for the relaxation of the excited state is the recombination of the electron with the hole, with fluorescence emission. The 4000-4700 $\AA$ blue bands, induced by both electron and positron bombardment, are gererated by this process. Transition to a metastable state through a radiationless intersystem crossing is another pathway for relaxation. The yellow band induced by electron bombardment may be due to this process. Optical transition from the 
metastable to the ground state appears to be slower than for the direct recombination process.

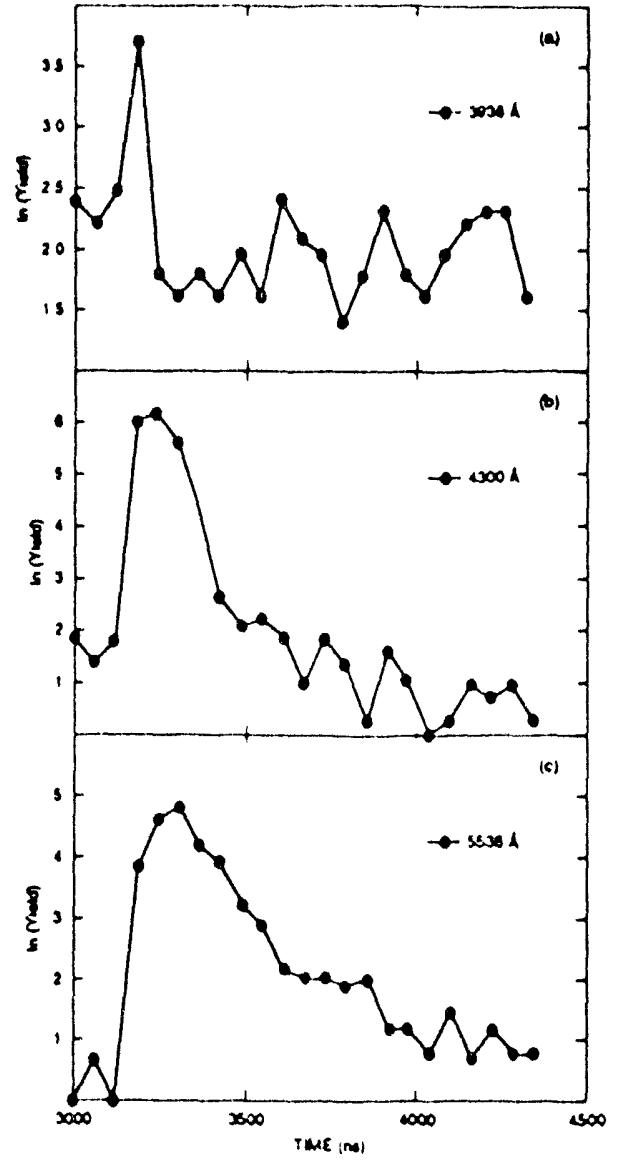

Fig. 4. Luminescence of the polymer as a function of time at (a) sub-blue band, (b) the blue band, and (c) the yellow band.

The yellow band appears to have a nonlinear relationship to the electron intensity. When the intensity of the electrons was reduced to approximately that of the positrons, the ratio of the yellow band intensity to the blue band intensity decreased. This suggests that the production of the metastable state is a twoelectron process, possibly involving the production of a short-lived defect by the first electron, followed by a trapping of an excited electron.

Positrons are able to remove molecular electrons from their ground states both by collisional excitation, the same as electrons, and by the annihilation process, which can remove bound electrons, leaving a hole, without the production of an excited electron. Transitions from impurity or surface state electrons to the single holes can produce light. The lower intensity blue band, below $4000 \AA$, is believed to relate this process.

More than $90 \%$ of the positrons will annihilate with the valance electrons as they are injected to a solid, while only a few percent of them annihilate with core electrons. Weiss and co-workers [3] have observed Auger electron emission induced by the annihilation of core electrons. It is very reasonable to predirt a high cross section for positron induced luminescence because this process is initiated by positron annihilation of valance electrons.

In summary, a clear difference between optical emissions from positron and electron bombardment of polymers has been observed. For positron bombardment, a blue band and a weak sub-blue band are observed, while the blue band and a yellow band are induced by electrons. The light emissions in the blue band are due to production of electron-hole pairs for both electron and positron cases. The yellow band is be due to metastable states generated by the intense electron beam. The sub-blue feature, induced only by positron bonibardment, is believed to be due to isolated electron holes, produced by annihilation.

This research was supported in part by an appointment (JX) to the Oak Ridge National Laboratory Postdoctoral Research Associates Program administered jointly by Oak Ridge National Laboratory and the Oak Ridge Institute for Science and Education and by the Chemical Sciences Division of the U. S. Department of Energy Office of Basic Energy Science, Contract No. DE-AC05-840R21400 with Martin Marietta Energy Systems, Inc.

[1] Jun Xu, L. D. Hulett, Jr., T. A. Lewis, and N. H. Tolk, Submitted to Applied surface science, (1994).

[2] R. S. Becker, Theory and Interpretation of Fluorescence and Phosphorescence, (Wiley Interscience, New York, 1969).

[3] A. Weiss, R. Mayer, M. Jibaly, C. Lei, D. Mehl, and K. G. Lynn, Phys. Rev. Lett, 61, 2245(1988). 

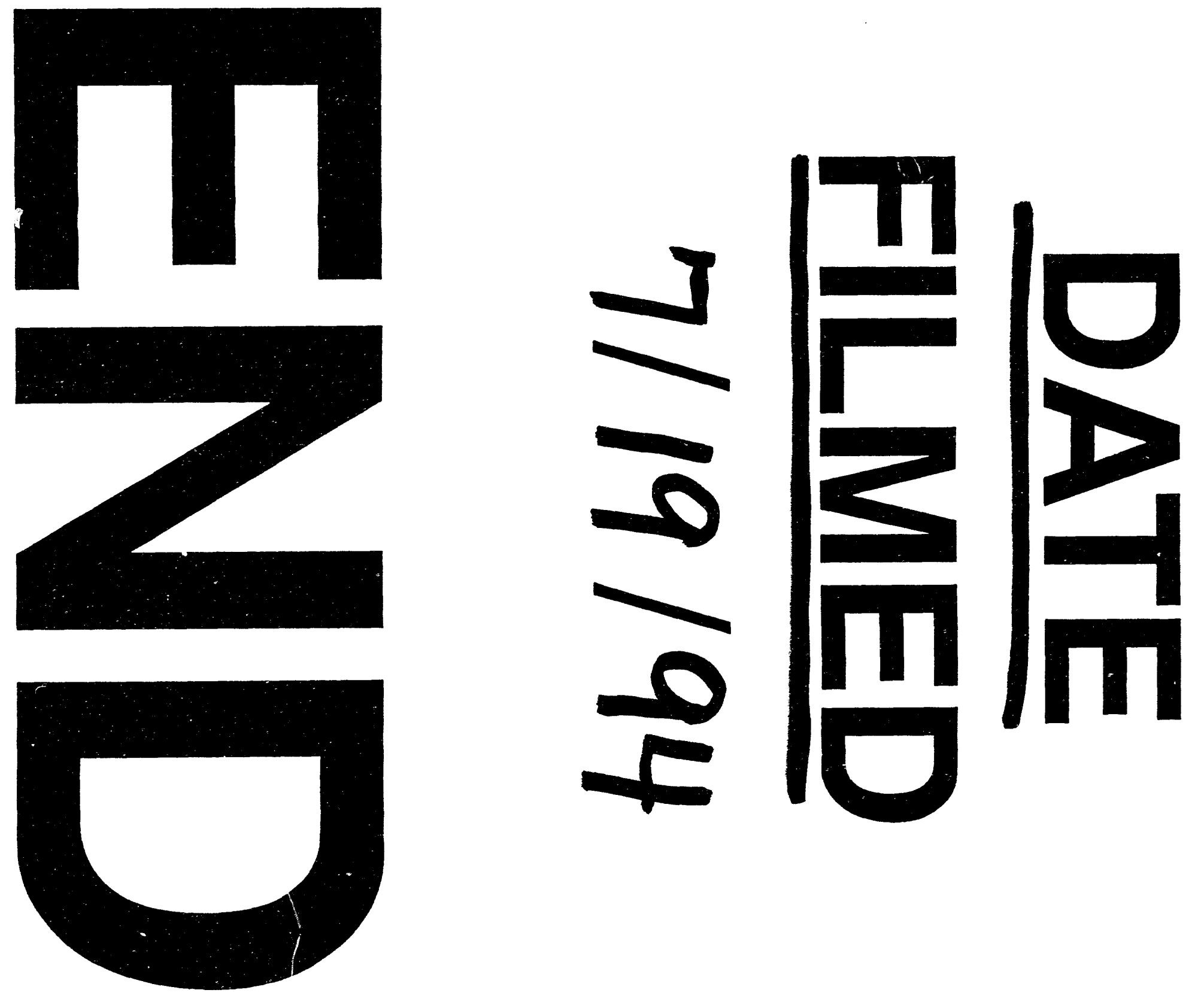
